\title{
Machine Learning Using Real-World and Translational Data to Improve Treatment Selection for NSCLC Patients Treated with Immunotherapy
}

\author{
Arsela Prelaj ${ }^{1,2, *}$, , Mattia Boeri ${ }^{3}{ }^{(0)}$, Alessandro Robuschi ${ }^{2}$, Roberto Ferrara ${ }^{1}$, Claudia Proto ${ }^{1}$, \\ Giuseppe Lo Russo ${ }^{1}$, Giulia Galli ${ }^{1}{ }^{1}$, Alessandro De Toma ${ }^{1}$, Marta Brambilla ${ }^{1}$, Mario Occhipinti ${ }^{1}$, \\ Sara Manglaviti ${ }^{1}$, Teresa Beninato ${ }^{1}$, Achille Bottiglieri ${ }^{1}$ (), Giacomo Massa ${ }^{1}$, Emma Zattarin ${ }^{1}$, Rosaria Gallucci ${ }^{1}$, \\ Edoardo Gregorio Galli ${ }^{1}$, Monica Ganzinelli ${ }^{1}$, Gabriella Sozzi ${ }^{3}$, Filippo G. M. de Braud ${ }^{1}$, \\ Marina Chiara Garassino ${ }^{1}$, Marcello Restelli ${ }^{2}\left[\right.$, Alessandra Laura Giulia Pedrocchi ${ }^{2,+}+\mathbb{C}$ and Francesco Trovo' ${ }^{2,+}(\mathbb{C}$
}

\section{check for}

updates

Citation: Prelaj, A.; Boeri, M.;

Robuschi, A.; Ferrara, R.; Proto, C.; Lo Russo, G.; Galli, G.; De Toma, A.; Brambilla, M.; Occhipinti, M.; et al. Machine Learning Using Real-World and Translational Data to Improve Treatment Selection for NSCLC Patients Treated with Immunotherapy. Cancers 2022, 14, 435. https://doi.org/ $10.3390 /$ cancers 14020435

Academic Editor: Charles B. Simone

Received: 19 December 2021

Accepted: 12 January 2022

Published: 16 January 2022

Publisher's Note: MDPI stays neutral with regard to jurisdictional claims in published maps and institutional affiliations.

Copyright: (C) 2022 by the authors. Licensee MDPI, Basel, Switzerland. This article is an open access article distributed under the terms and conditions of the Creative Commons Attribution (CC BY) license (https:// creativecommons.org/licenses/by/ $4.0 /)$.
1 Medical Oncology Department, Fondazione IRCCS Istituto Nazionale Tumori, 20133 Milan, Italy; roberto.ferrara@istitutotumori.mi.it (R.F.); claudia.proto@istitutotumori.mi.it (C.P.); giuseppe.lorusso@istitutotumori.mi.it (G.L.R.); giulia.galli@istitutotumori.mi.it (G.G.); alessandro.detoma@istitutotumori.mi.it (A.D.T.); marta.brambilla2@istitutotumori.mi.it (M.B.); mario.occhipinti@istitutotumori.mi.it (M.O.); sara.manglaviti@istitutotumori.mi.it (S.M.); teresa.beninato@istitutotumori.mi.it (T.B.); achille.bottiglieri@istitutotumori.mi.it (A.B.); giacomo.massa@istitutotumori.mi.it (G.M.); emma.zattarin@istitutotumori.mi.it (E.Z.); rosaria.gallucci@istitutotumori.mi.it (R.G.); edoardogregorio.galli@studenti.unimi.it (E.G.G.); monica.ganzinelli@istitutotumori.mi.it (M.G.); filippo.debraud@istitutotumori.mi.it (F.G.M.d.B.); marina.garassino@istitutotumori.mi.it (M.C.G.)

2 Department of Electronics, Information and Bioengineering, Politecnico di Milano, 20133 Milan, Italy; alessandro.robuschi@polimi.it (A.R.); marcello.restelli@polimi.it (M.R.); alessandra.pedrocchi@polimi.it (A.L.G.P.); francesco1.trovo@polimi.it (F.T.)

3 Tumor Genomics Unit, Department of Research, Fondazione IRCCS Istituto Nazionale dei Tumori, 20133 Milan, Italy; mattia.boeri@istitutotumori.mi.it (M.B.); gabriella.sozzi@istitutotumori.mi.it (G.S.)

* Correspondence: arsela.prelaj@istitutotumori.mi.it

+ These authors contributed equally to this work.

Simple Summary: In this paper, the authors show that artificial intelligence (AI) and machine learning (ML) are useful approaches to integrate multifactorial data and helpful for personalized prediction. In detail, compared to PD-L1 for advanced non-small cell lung cancer (NSCLC), ML tools predicted better responder (R) and non-responder (NR) patients to immunotherapy (IO). It was also able to indirectly foresee OS and PFS of R and NR patients. Given the high incidence of NSCLC, and the absence of reliable biomarkers to predict the response to IO other than PD-L1, the authors believe this research may be of great interest to anyone involved in thoracic oncology. Furthermore, given the growing interest from the scientific community in $\mathrm{AI}$ and $\mathrm{ML}$, the authors believe that this manuscript could represent a fascinating topic to anyone who needs to exploit the enormous potential of these tools in the treatment of cancer.

Abstract: (1) Background: In advanced non-small cell lung cancer (aNSCLC), programmed death ligand 1 (PD-L1) remains the only biomarker for candidate patients to immunotherapy (IO). This study aimed at using artificial intelligence (AI) and machine learning (ML) tools to improve response and efficacy predictions in aNSCLC patients treated with IO. (2) Methods: Real world data and the blood microRNA signature classifier (MSC) were used. Patients were divided into responders (R) and non-responders (NR) to determine if the overall survival of the patients was likely to be shorter or longer than 24 months from baseline IO. (3) Results: One-hundred sixty-four out of 200 patients (i.e., only those ones with PD-L1 data available) were considered in the model, 73 (44.5\%) were R and $91(55.5 \%)$ NR. Overall, the best model was the linear regression (RL) and included 5 features. The model predicting R/NR of patients achieved accuracy ACC $=0.756, \mathrm{~F} 1$ score F1 $=0.722$, and area under the ROC curve AUC $=0.82$. LR was also the best-performing model in predicting patients with long survival (24 months OS), achieving $\mathrm{ACC}=0.839, \mathrm{~F} 1=0.908$, and $\mathrm{AUC}=0.87$. (4) Conclusions: 
The results suggest that the integration of multifactorial data provided by ML techniques is a useful tool to select NSCLC patients as candidates for IO.

Keywords: non-small cell lung cancer; immunotherapy; biomarker; artificial intelligence; machine learning

\section{Introduction}

Lung cancer is the leading cancer-related death worldwide with around 470,000 new cases and 390,000 deaths in Europe. Non-small cell lung cancer (NSCLC) is the most common histology for around $85 \%$ [1]. Until 2015, the median OS of patients with metastatic NSCLC was around 12 months [2]. The advent of immunotherapy (IO) has radically changed the treatment paradigm of many cancers including NSCLC, prolonging survival of metastatic patients from 12 to a median of around 24 months [2]. Some patients that better respond to IO reached longer survival of up to or more than 5 years [3]. However, only $30-50 \%$ of patients will benefit from IO in the long term [4-6].

Currently in clinical practice, programmed death-ligand 1 (PD-L1) is the only biomarker used to predict IO response. However, its predictive performance is not satisfactory (around 30-50\%) [7]. Beyond PD-L1, several other biomarkers have been identified and used to profile patient prediction, including tumor mutational burden (TMB) [8], tumor microenvironment (TME) [9], microRNA (miRNA) [10], immune gene signatures [11], gut microbiome [12], radiomics [13], and baseline clinical features or their combination in different scores $[14,15]$.

Indeed, it is implausible that a single biomarker is able to profile prediction or prognosis with high accuracy, since the immune system displays dynamic complexity when interacting with its TME. To handle the density of the available data, artificial intelligence (AI) frameworks and, more specifically, machine learning (ML) techniques, provide efficient, pioneering, and theoretically sound approaches to construct decision-making tools providing individualized prediction [16].

Among molecular biomarkers, the plasma microRNA signature classifier (MSC), reflecting an immunosuppressive host status, was here considered [10]. It was previously trained in lung cancer screening cohorts to evaluate the individual risk to develop the aggressive form of the disease $[17,18]$. More recently, the MSC prognostic value was also validated in advanced NSCLC patients treated with single agent IO [19], and its combination with different clinical scores confirmed its independence from other prognostic features in this setting [20].

This study aimed to integrate real-world data and the MSC test to develop a machine learning algorithm to predict response to and efficacy of IO in NSCLC patients. The study also investigated the role of the MSC test and its added value to the algorithm prediction capability, given that this latter test is costly and still not included in standard clinical practice as a predictive/prognostic biomarker.

\section{Materials and Methods}

\subsection{Study Population}

From July 2015 to November 2020, we conducted a prospective observational study (Apollo, INT 22_15) enrolling 200 consecutive aNSCLC patients receiving single-agent anti-PD-(L)-1 inhibitors in first- $(n=70)$ or second- and further-line therapy $(n=130)$. Complete real-world data and whole blood samples were collected as per clinical practice. The MSC test was prospectively assessed in plasma samples collected at baseline IO.

Inclusion criteria were the following: cytological/histological diagnosis of advanced NSCLC, patients (relapsed or stage IIIB to IV) that had received at least one infusion of first- or further-line single-agent IO. Patients without baseline IO MSC test information were excluded from the study. 
This prospective study was conducted at Fondazione IRCCS Istituto Nazionale Tumori of Milan in Italy in collaboration with Politecnico di Milano, for the data analytics. This study was approved by the ethical committee of Fondazione IRCCS Istituto Nazionale Tumori of Milan, and all included patients signed informed consent prior to plasma and data collection in accordance with the Declaration of Helsinki, Good Clinical Practice and local ethical guidelines.

\subsection{Real World Data Collection: Clinical, Blood, and Tissue Data}

For this study, demographic, medical history, tumor stage, PD-L1 (PD-L1 testing was mostly carried out using the PD-L1 IHC 22C3), molecular and radiological data, concomitant medications, treatment responses, and survival follow-up were collected and integrated to develop e new predictive model of IO response and efficacy in NSCLC.

\subsection{Omic Collection: MSC Blood Test}

Whole blood was collected in $10 \mathrm{~mL}$ K2EDTA Vacutainer tubes, and the plasma separated by two centrifugation steps. Total RNA was extracted from $200 \mu \mathrm{L}$ plasma samples. MicroRNA expression was determined by quantitative reverse transcription PCR (RT-qPCR) as previously described $[19,21]$.

The MSC algorithm using 24 miRNAs defined four different classes of risk: low (L) intermediate (I) and high $(\mathrm{H})$ risk [18] and highly hemolyzed (E). The fourth category $\mathrm{E}$, thus not analyzable plasma samples, due to the unspecific release of miRNAs in the presence of blood cell lyses, was included [10] (Figure 1). The 24 miRNAs were hsa-miR101-3p, hsa-miR-106a-5p, hsa-miR-126-3p, hsa-miR-133a-3p, hsa-miR-140-3p, hsa-miR-1405p, hsa-miR-142-3p, hsa-miR-145-5p, hsa-miR-148b-3p, hsa-miR-15b-5p, hsa-miR-16-5p, hsa-miR-17-5p, hsa-miR-197-3p, hsa-miR-19b-3p, hsa-miR-21-5p, hsa-miR-221-3p, hsamiR-28-3p, hsa-miR-30b-5p, hsa-miR-30c-5p, hsa-miR-320a, hsa-miR-451a, hsa-miR-486-5p, hsa-miR-660-5p, and hsa-miR-92a-3p. Patients with this category were previously observed to have an intermediate prognosis between patients with $\mathrm{H}$ and I risk [20].

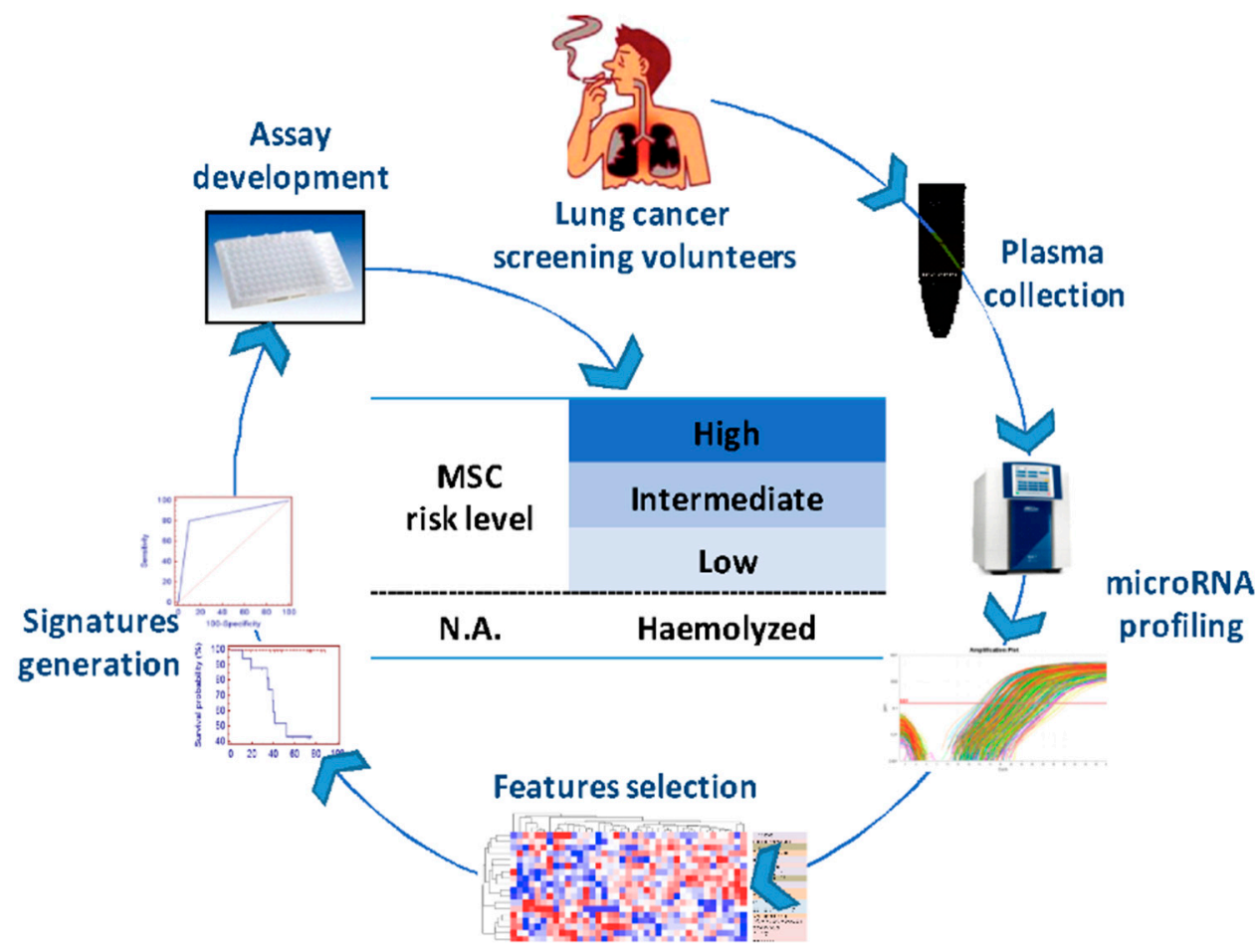

Figure 1. Development of the plasma MSC test using 24 miRNA and 4 risk groups. MSC: microRNA signature classifier; N.A.: not analyzable. 


\subsection{Treatment Administration}

IO was administered intravenously (IV) as monotherapy. Nivolumab was administered initially at a dose of $3 \mathrm{mg} / \mathrm{kg}$ and later, from May 2018 in Italy, at a fixed dose of $240 \mathrm{mg}$ every 2 weeks (w). Pembrolizumab was administered at a fixed dose of $200 \mathrm{mg}$ as first line and at a dose of $2 \mathrm{mg} / \mathrm{kg}$ every 3 weeks in second or third-line setting. Atezolizumab was administered at a fixed dose of $1200 \mathrm{mg}$ every 3 weeks, and durvalumab at a dose of $10 \mathrm{mg} / \mathrm{kg}$ every 2 weeks.

Therapy was continued until progressive disease (PD), intolerable toxicity, withdrawal or death from any cause. Treatment beyond PD was allowed if there was a clinical benefit according to clinician's decision.

\subsection{Radiological Response Evaluation}

Baseline radiological evaluations included a baseline total body computed tomography (TB-CT) scan, subsequently performed every 3-4 cycles or every 9-12 weeks as per standard of care, or whenever progression was clinically suspected. Six categories of radiological response were taken into consideration in this study to assess tumor response. Four of them (standard categories) were included in Response Evaluation Criteria in Solid Tumors (RECIST1.1): complete response (CR), partial response (PR), stable disease (SD), and progressive disease (PD). Two additional categories were included: hyper progression disease (HPD), an atypical pattern of response to single-agent IO (an acceleration of progression compared to the natural history of the disease) as defined by Ferrara et al. [22] and Lo Russo et al. [23], and eventually, not evaluable (NE) as the sixth category, comprising those patients who died due to PD before the first radiological evaluation.

\section{Statistics and AI Methodology}

Figure 2 reports an outline of all the methodologies applied for data analyses.

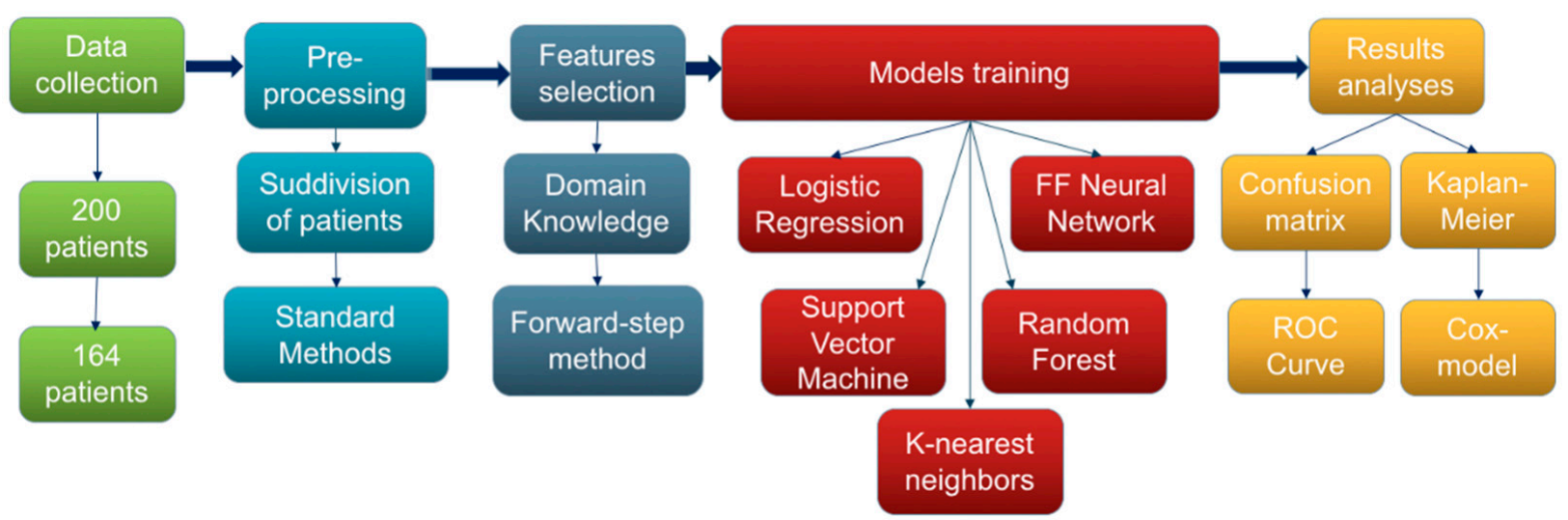

Figure 2. Process and methods used in this study.

\subsection{Statistical Analysis}

Out of the 200 patients included in the present study having available PD-L1 expression data, 164 patients were used as the dataset for the ML algorithms, since PD-L1 was the only predictor used in clinical practice. Conversely, all 200 patients were included in the survival analysis. The first endpoint of the study was prediction of responder $(R)$ and non-responder (NR) patients. The R group included patients who obtained a CR, PR, or SD as per RECIST 1.1, while the NR group included those patients who obtained a PD per RECIST1.1., or an HPD or NE response (as described above).

Other endpoints were at 24-months overall survival (OS), median progression-free survival (mPFS), and median OS (mOS). mOS was measured from the starting date of IO therapy until death, or last follow-up. mPFS was calculated from the starting date of IO until PD or death due to any cause, or last follow-up visit for alive patients without PD. 
Kaplan-Meier was used to calculate MPFS and mOS with their 95\% confidence interval, and to generate survival curves. Cox's proportional hazards model was used to calculate the hazard ratio (HR) between R and NR groups according to OS and PFS.

\subsection{Machine Learning Methods}

After data collection, descriptive analysis and data processing were performed. A first step consisted of the selection of a set of 21 features determined to be the most relevant based on the published literature on NSCLC patients treated with IO and clinician experience. Finally, in the case where a pair of features showed a linear correlation higher than 0.8 , we removed one of them, as customary in ML studies. The result was the set of $\mathrm{M}=15$ most relevant features, provided in Table 1.

Table 1. Features selected based on literature review and clinician experience, and keeping only one of the variables in a pair showing linear correlation $>0.8$.

\begin{tabular}{cc}
\hline Feature Classes & Features \\
\hline Clinical features & Age, sex, smoker/non-smoker, packs per year, ECOG \\
\hline Laboratory exams & NLR * NLR4, LDH \\
\hline Tumor features & PD-L1, histology (adenocarcinoma, squamous, other) \\
\hline Radiological & Metastatic sites (liver, brain, bone) \\
\hline Treatment features & IO line (first or further line) \\
\hline Omic features & MSC test \\
\hline${ }^{*}$ NLR was used both as a continuous variable or binary variable with cut-off 4.
\end{tabular}

The problem of predicting $\mathrm{R}$ and NR was modelled as a binary classification problem, where we wanted to learn an approximation $\mathrm{f}\left(\mathrm{x} \_\mathrm{i}\right)$ of the real relationship $\mathrm{y}=\left(\mathrm{x} \_\mathrm{i}\right)$ between the $i$-th patient's feature vector $x_{-} i$ and the response $y_{-} i \in\{0,1\}$, where a patient has $y_{-} i=0$ for $\mathrm{NR}$, and $\mathrm{y}_{-} \mathrm{i}=1$ for $\mathrm{R}$. The same modelling was applied to the problem of estimating survival at 24 months, i.e., a patient has $y_{-} i=0$ for those patients with OS less than 24 months and y_i = 1 for those with more than 24 months. Data corresponding to the 40 alive patients with less than 24 months were excluded from this second analysis.

A set of appropriate techniques from the ML literature were selected to perform the above-mentioned classification task. More specifically, feedforward neural network (FFNN), logistic regression (LR), K-nearest neighbors (K-NN), support vector machines (SVM), and random forest (RF) were tested. A feature selection approach to select the proper subset of the original $\mathrm{M}$ features appropriate for each method were applied. More specifically, a forward feature selection using the AIC criterion as metric to select the most appropriate set of features for each method and the best method were used. The 5 -fold cross-validation ACC and F1 scores for the analyzed methods, as well as the leave one out AUC, with the corresponding $95 \%$ confidence intervals were computed using the bootstrap approach (in brackets).

The procedure was implemented in Matlab, and the code performing all of the ML procedures is available at https://trovo.faculty.polimi.it/downloads.html (accessed on 10 October 2021) [24].

\section{Results}

\subsection{Patients' Characteristics}

Two hundred NSCLC patients treated with anti-PD-(L)-1 in first or further-line therapy were included in the survival analysis. Most patients were male (65\%) and smokers (79.5\%), median age was 67 years (range $60-74$ years), and $38 \%$ of patients were older than 70 years. PD-L1 was $\geq 50 \%$ in $53(26.5 \%), 1-49 \%$ in $59(29.5 \%),<1 \%$ in $52(26 \%)$ and unknown in $36(18 \%)$ patients. Median ECOG-PS was 1 (range 0-1) with an ECOG PS 2 in $14.5 \%$ of patients. All patients had a histological diagnosis of NSCLC (77\% non-squamous and $23 \%$ 
squamous) and were epidermal growth factor receptor (EGFR) non-mutated and anaplastic lymphoma kinase gene (ALK) non-translocated. At baseline IO, liver metastases were present in 35 (17.5\%) of patients. More than one-third of patients (35\%) received IO in first line, while the remaining patients received anti-PD-(L)-1 therapy in further lines. Overall, $40(20 \%)$ patients were $\mathrm{H}, 65(32.5 \%)$ were $\mathrm{I}$, and $54(27 \%)$ were L according to MSC risk level. On the other hand, 41 (20.5\%) patients were E and thus not analyzable.

One-hundred sixty-four patients were enrolled in this study, and patients were divided into two major groups: 73 belonged to the R group (CR, PR, or SD), and 91 to the NR group (PD, HPD, or NE).

\subsection{Predicting Responder and Non-Responder Patients}

Table 2 presents the results of the feature selection procedure. The best model turned out to be the logistic regression, which included 5 features: ECOG performance status, IO line of therapy, the neutrophil-to-lymphocyte ratio (NLR), the MSC test, and PD-L1. The importance of the variables was provided directly by the magnitude (absolute value) of the coefficient obtained by the logistic regression. More specifically, in order of importance for the LR, the parameter vectors learned by LR were $\mathrm{w}=1.058$ (NLR), 0.71 (IO line), 0.692 (ECOG), 0.566 (MSC), and -0.471 (PD-L1 > 50\%). This showed how an increase in one of the first four features was negatively correlated with patients' response, and conversely, how the increase in the PD-L1 value correlated positively with response (the only negative coefficient).

Table 2. Features selected for the different models and corresponding performances.

\begin{tabular}{cccccc}
\hline ML Model & Selected Features & AIC & ACC & F1 & AUC \\
\hline LR & $\begin{array}{c}\text { ECOG, IOLine, NLR, } \\
\text { MSC, PD-L1 }\end{array}$ & 132.5 & 0.756 & 0.722 & $\begin{array}{c}0.83 \\
(0.76-0.88)\end{array}$ \\
\hline FFNN & $\begin{array}{c}\text { NLR, IOLine, MSC, } \\
\text { LDH, ECOG, PackYear }\end{array}$ & 137.2 & 0.732 & 0.686 & $\begin{array}{c}0.80 \\
(0.73-0.86)\end{array}$ \\
\hline K-NN & $\begin{array}{c}\text { NLR, IOLine, ECOG, } \\
\text { MSC, NLR4 }\end{array}$ & 137.4 & 0.726 & 0.667 & $\begin{array}{c}0.81 \\
(0.74-0.87)\end{array}$ \\
\hline SVM & $\begin{array}{c}\text { ECOG, IOLine, NLR, } \\
\text { MSC, PD-L1 }\end{array}$ & 134.5 & 0.738 & 0.703 & $\begin{array}{c}0.83 \\
\text { RF }\end{array}$ \\
\hline $\begin{array}{c}\text { NLR, IOLine, ECOG, } \\
\text { Age, MSC }\end{array}$ & 135.5 & 0.701 & 0.657 & $\begin{array}{c}0.82 \\
(0.73-0.87)\end{array}$ \\
\hline
\end{tabular}

For each model, the confusion matrix is presented in Figure 3 to show their performances in terms of true/false positives/negatives.

Logistic regression as the best model achieved an ACC $=0.756, \mathrm{~F} 1=0.722$, and AUC $=0.83$. PD-L1 alone had an ACC $=0.655$ (whose performances are illustrated by the red circle in Figure 4). We also evaluated the accuracy of the LR models excluding PD-L1, MSC, and both PD-L1 and MSC from the models, i.e., considering only clinical features. Moreover, we excluded the ECOG, being the only physician-dependent feature. The results of these models are shown in Table 3, and the ROC curves are provided in Figure A1. Removing PD-L1, the accuracy of the corresponding model decreased to ACC $=0.726$, confirming the high importance of this feature, as reported in the literature. Removing the MSC from the feature decreased the accuracy to ACC $=0.750$, suggesting that the predictive power of this index was less impactful than PD-L1. Removing both from the data yielded an $\mathrm{ACC}=0.707$. 


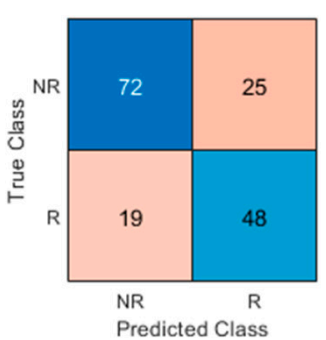

(a) $\mathrm{NN}$

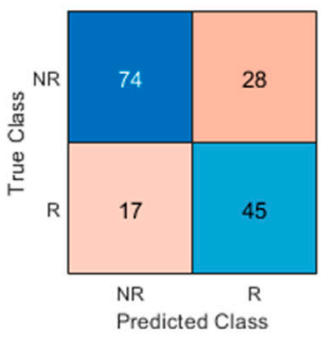

(c) K-NN

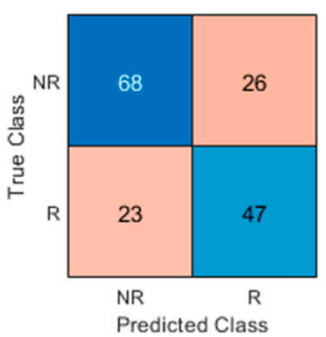

(e) RF

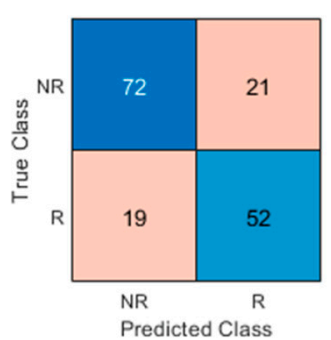

(b) LR

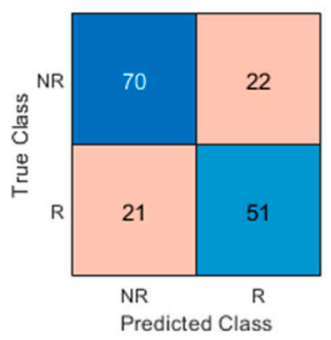

(d) SVM

Figure 3. Confusion matrix for the analyzed ML models for responders (R) and non-responders (NR) for ML model algorithms (a) NN, (b) LR, (c) K-NN, (d) SVM and (e) RF.

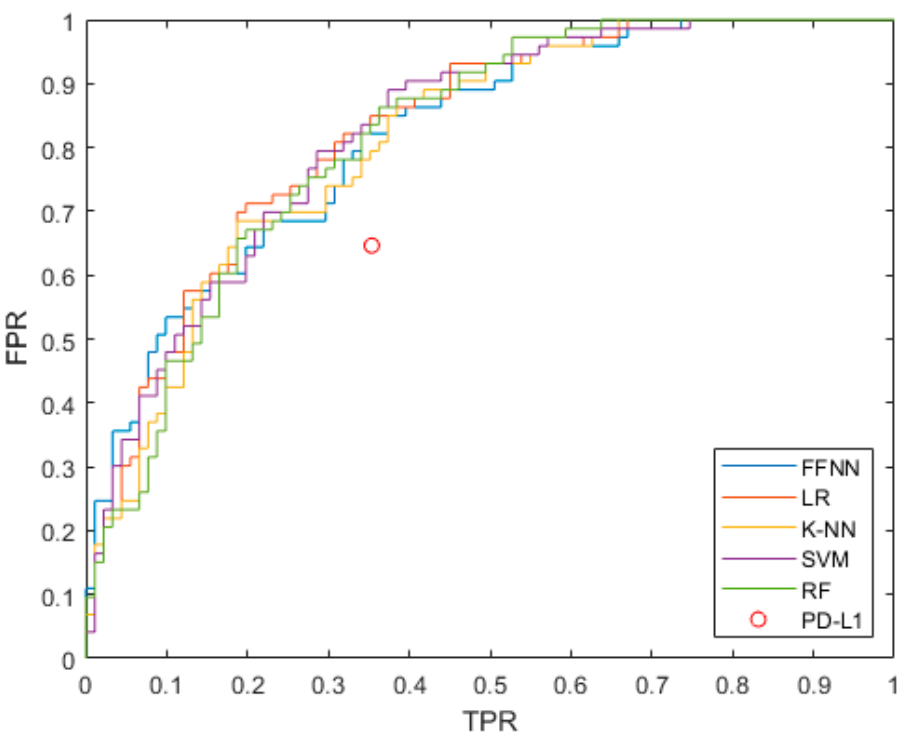

Figure 4. ROC curves (true positive rate (TPR) vs. false positive rate (FPR)) for the analyzed ML models. The performance of PD-L1 is represented as a red circle. As suggested by the AUC confidence intervals, there is no method that outperforms the others significantly. 
Table 3. Performances of the LR method when some of the features are removed from the initial pool of available ones.

\begin{tabular}{ccccc}
\hline Initial Feature Set & Selected Features & ACC & F1 & AUC \\
\hline All & $\begin{array}{c}\text { ECOG, IOLine, NLR, } \\
\text { MSC, PD-L1 }\end{array}$ & 0.756 & 0.722 & $0.83(0.76-0.88)$ \\
\hline No PD-L1 & ECOG, IOLine, NLR, MSC & 0.726 & 0.696 & $0.82(0.75-0.88)$ \\
\hline NO MSC & ECOG, IOLine, NLR, PD-L1, Age & 0.750 & 0.709 & $0.81(0.74-0.87$ \\
\hline NO PD-L1 and MSC & ECOG, IOLine, NLR, Age & 0.707 & 0.662 & $0.80(0.73-0.86)$ \\
\hline NO ECOG & IOLine, NLR, MSC, PD-L1 & 0.726 & 0.690 & $0.80(0.73-0.87)$ \\
\hline
\end{tabular}

Finally, removing the ECOG decreased the accuracy of the LR model to ACC $=0.726$; therefore, the importance of the physician clinical evaluation was comparable to PD-L1 in the prediction. These findings were confirmed by the values of the F1 score and the average AUC (Table 3). The ROC curve obtained by the leave-one-out method is presented in Figure 4.

\subsection{Survival Analysis According to PFS and OS}

Since good results were obtained in classifying patients as responders and nonresponders, it was also possible to estimate the mOS and mPFS of these patients using KM curves, as shown in Figure 5a,b. At data cut-off (November 2020), mOS was 10.1 months for all patients. Median PFS for the R and NR groups was 11.4 vs. 1.8 months (HR 0.095, $95 \%$ CI $0.062-0.114, p<0.0001$ ), and the median OS, 38.5 vs. 3.8 months (HR $0.123,95 \%$ CI $0.079-0.193, p<0.0001)$. Appendix A contains all of the Kaplan-Meier curves separately according to first and further-line therapy in R and NR patients, respectively (Figure A2).
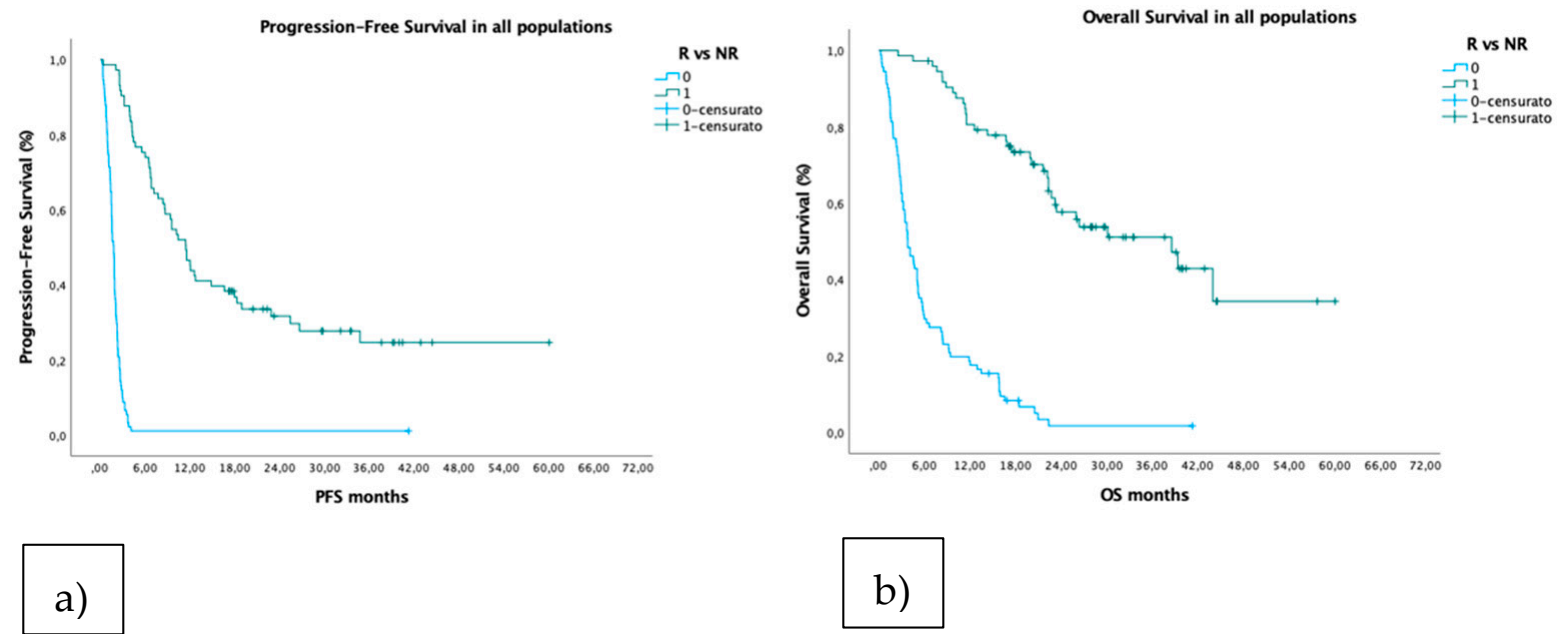

a)

Figure 5. Kaplan-Meier according to PFS (a) and OS (b) curves divided by R (red curves) and NR (blue curves) groups.

\subsection{Predicting Long-Survival Patients ( $\geq 24-$ Months OS)}

To predict long-survival ( $\geq 24$-months OS) patients, another ML binary classification analysis was performed.

Because we were solving a different classification model, we needed to reconsider the use of the above-mentioned methods from scratch. Table 4 lists all of the procedures for feature selection. Even in this case, the LR method proved to be the most promising according to the AIC criterion. It achieved an ACC $=0.855, \mathrm{~F} 1=0.908$, and AUC $=0.87$. The features included in the model were ECOG, histology, NLR, and IO line. 
Table 4. Features selected for the different models and corresponding performances for the task of predicting the long- survival patients.

\begin{tabular}{cccccc}
\hline ML Model & Selected Features & AIC & ACC & F1 & AUC \\
\hline LR & $\begin{array}{c}\text { ECOG, Histology, } \\
\text { NLR, IOLine }\end{array}$ & 58.1 & 0.855 & 0.917 & $\begin{array}{c}0.89 \\
(0.80-0.94)\end{array}$ \\
\hline FFNN & $\begin{array}{c}\text { Histology, NLR, } \\
\text { PD-L1, NLR4 }\end{array}$ & 61.4 & 0.839 & 0.908 & $\begin{array}{c}0.87 \\
(0.78-0.92)\end{array}$ \\
\hline K-NN & NLR, PD-L1, Histology & 60.6 & 0.847 & 0.916 & $\begin{array}{c}0.88 \\
(0.80-0.93)\end{array}$ \\
\hline SVM & $\begin{array}{c}\text { Age, Histology, MSC, } \\
\text { ECOG, PD-L1, NLR }\end{array}$ & 63.2 & 0.847 & 0.913 & $\begin{array}{c}0.90 \\
(0.83-0.94)\end{array}$ \\
\hline RF & NLR, PD-L1 & 63.8 & 0.847 & 0.917 & $\begin{array}{c}0.83 \\
(0.74-0.89)\end{array}$ \\
\hline
\end{tabular}

The ROC curves computed using the leave-one-out approach are provided in Figure A3.

\section{Discussion}

The use of AI is attracting great interest in the medical field and, in particular, in oncology. The recent literature contains a wide range of publications regarding the use of AI applied to NSCLC, especially focusing on real-world data, genomics, circulomics, and radiomics. In our study, we aimed to find an algorithm to predict response to and efficacy of IO using real-world data (i.e., clinical, tumor, and treatment data) and translational data (i.e., the results of the MSC test). Combining the current medical literature, clinical experience of physicians, and ML tools, we developed an algorithm including five important features discriminating between $\mathrm{R}$ and NR patients with good accuracy ( $\mathrm{ACC}=0.756, \mathrm{~F} 1=0.722$, and AUC $=0.83$ ). The model achieved significantly better results compared to PD-L1 prediction value alone, which is the only biomarker currently used by physicians in clinical practice to select NSCLC patients for IO with an accuracy of ACC $=0.655$ on the analyzed dataset. To determine whether the algorithm maintained its accuracy using only real-world data, we decided to exclude the PD-L1 from the model features. In this case, the accuracy of the model decreased, suggesting that even if the PD-L1 alone is not enough to provide an effective response prediction, it remains an essential feature for IO prediction to be used in clinical practice. We did the same with the MSC, since this test is an expensive and time-consuming exam, and, therefore, its introduction in clinical practice needs to be justified. When we excluded the MSC from the model, the model accuracy decreased, albeit by less than in the case of PD-L1 exclusion, again suggesting that the MSC has a role in our model. We also tested the model removing the patient's ECOG, which is a physician-dependent value, and the results demonstrated a significant impact, analogous to PD-L1. Since the model was able to discriminate between R and NR groups, we were also able to indirectly predict the PFS and OS of these patients.

With a binary classification approach, we provided a method to identify and predict those patients with long OS ( $\geq 24$-months OS). Even in this case, the use of ML techniques showed a significant improvement over the use of PD-L1 (ACC $=0.855, \mathrm{~F} 1=0.908$, and $\mathrm{AUC}=0.87$ vs. $\mathrm{ACC}=0.734$ ).

Various papers have been recently published to address the same unmet clinical need not only in NSCLC but also in other different cancer types.

Radiomics features are frequently used to predict IO response in NSCLC patients. In the study by $\mathrm{He}$ et al. [25] with a dual propose, radiomics were applied to build a TMB signature. CT images were used to discriminate between high-TMB and low-TMB in 327 patients. The model was then applied to the IO of 123 patients' dataset to evaluate risk stratification. The TMB radiomic signature reached an AUC of 0.74 [5]. The prediction was slightly lower compared to our study, probably indicating that the clinical features 
and patients' presentations have comparably high relevance as tumor features and that it is important to consider them in the model.

Khorrami et al. [26] compared changes ("delta") in the radiomic texture of CT scan patterns (139 patients) and associated them with tumor-infiltrating lymphocyte (TIL) density in diagnostic biopsies from 36 patients. A linear discriminant analysis classifier yielded an AUC of $0.88 \pm 0.08$ in distinguishing $\mathrm{R}$ from NR patients when CT scan features were combined with TIL density. However, 36 patients were included in this coupled analysis, and even if our study achieved a lower AUC, our model included four real-world datasets that were easier to be obtained compared to radiomics and TIL analysis.

Yang et al. [27] used 200 patients to develop a deep learning (DL) model integrating different data sources (serial radiomics, CT scans, laboratory and baseline clinical data) to identify R and NR subgroups to IO in NSCLC patients. The model reported an AUC of 0.80 (95\%CI: 0.74-0.86), showing a smaller than expected value when compared to ours (AUC 0.82). A very interesting study called DeePaN [28] used a deep patient graph convolutional network to investigate the IO benefit in NSCLC patients. By integrating real-world data (age, sex, race, histology, stage, ECOG score, smoking status and previous treatment, blood analyses) and genomics in 1937 patients, the algorithm was able to divide patients into two different subgroups: beneficial and non-beneficial patients with an mOS of 20.35 and 9.42 months, respectively. Even though our sample was smaller, our model was able to predict survival and response with comparable results. The model also demonstrated the positive role of TMB and KRAS mutation in IO patients [28]. The study by Tian et al. [29] had a dual purpose: first, to predict a PD-L1 signature (PD-L1ES) using CT images (in 939 patients), and second, to predict IO response in NSCLC patients combining PD-L1ES and clinical features (in 77 patients). PD-L1ES was able to distinguish patients with a better PFS compared to those with a lower PFS. However, results of the combined model (PD-L1ES and clinical data) were superior to both the clinical and PD-L1ES models alone [29]. Our study also confirmed the importance of PD-L1 and the value it added to clinical features.

The development and validation of a 12-gene immune relevant prognostic signature for lung adenocarcinoma through ML strategies was investigated in 954 patients to predict IO. From a discovery dataset of 204 observations including microarray data of gene expression of 1811 genes, Cox regression was used to decrease the number of features to 336. Random forest was then used to extract the final 12 selected genes used to compute the risk score. Patients were classified into high- or low-score with an AUC of 0.854 (95\%CI $=0.79-0.92)$. Patients with a high-risk score experienced lower survival comparing to those with the low-risk score $(\mathrm{HR}=10.6,95 \% \mathrm{CI}=3.21-34.95, p<0.001)$ [30].

Independently from $\mathrm{IO}, \mathrm{ML}$ and DL techniques are now used in research to predict NSCLC prognosis for patients treated with different therapies to better address precision medicine; however, these techniques are still far from their introduction in clinical practice. An interesting study used DL to implement OS prediction in NSCLC patients by integrating microarray and clinical data. A list of 15 relevant genes was built using seven known relevant biomarker genes and eight other less-known genes. Expression data on the 15 genes and the clinical data were combined and used to develop an integrative deep NN predicting the 5-year survival status of NSCLC patients with high accuracy (AUC: 0.8163, accuracy: $75.44 \%$ ); these data were consistent and comparable with our results [31]. Another study developed an algorithm to predict NSCLC survival time in 1000 patients treated with different types of therapies. Thirteen features were included in the algorithm, e.g., number of primaries, tumor size, age, and stage. Random forest was the best model to predict short-term survival period ( $<6$ months) [32].

Finally, IO biomarker prediction, as we mentioned above, is an unmet clinical need also for other cancer types. In fact, as in NSCLC, various efforts have been made to find predictive biomarkers of IO response using ML or DL methodology in other cancers. An interesting report on melanoma patients integrated histologic data and clinical data to predict IO response. The algorithm consisted of a segmentation classifier that took as input 
the whole slide image of the patient (hematoxylin and eosin tissue). These results were then combined through a multivariable logistic regression with clinical characteristics such as age, gender, histologic subtype, etc. The classifier accurately stratified patients into high versus low risk for disease progression with an $\mathrm{AUC}=0.80$ [33].

Gene expression data were used to separate gastric metastatic cancer patients into durable clinical benefit (DCB) and non-durable clinical benefit (NDCB) groups considering a training dataset of 25 (DCB) plus 45 (NDCB) and a validation cohort of 9 (DCB) plus 15 (NDCB), obtaining an accuracy of $\mathrm{ACC}=0.857$ in the validation cohort [34].

Lastly, in another work regarding IO prediction in bladder cancer, CT-scans were used to develop an ML model according to the RECIST methodology, and the ROIs were processed to extract radiomic features. Considering a dataset of 43 subjects, the model reached an accuracy of ACC $=0.861$ [35].

Our study had various limitations: firstly, the limited sample size. Secondly, we did not use radiomic features in our study, and no genomic data were included except the unique molecular data requested for standard of care.

Many studies have sought to extract more information from imaging (radiomics) and genomic data. Radiomics is a very important frontier but still in an early phase, and more time will be needed to include it in clinical practice. The same may be said for genomics. The approach adopted in this paper used routine information from imaging (e.g., RECIST) as well as real-world genetic data that had already been investigated as per standard of care, both of which added to the clinical information and enabled better extraction of predictive multifactorial information. These data can also be less expensive and easier to collect.

\section{Conclusions}

In conclusion, the results suggest that the data integration provided by AI techniques is a good tool to improve prediction for NSCLC patients treated with IO. More specifically, the model showed that higher ECOG, NLR value, IO line, and MSC test level correlated negatively with the response to IO therapy, whereas conversely, higher PD-L1 correlated positively with the response. It also confirmed that PD-L1 and MSC were relevant biomarkers to improve the accuracy of the model. Moreover, considering the difference in survival among $\mathrm{R}$ and NR groups, these results suggest that the model could also be used to indirectly predict survival (PFS and OS).

Finally, a second binary model was able to identify long survival patients with high accuracy.

Author Contributions: A.P.: conceptualization, investigation, methodology, project administration, supervision, writing — original draft, writing—review and editing; M.B. (Mattia Boeri): data curation, formal analysis, methodology, resources; A.R.: data curation, formal analysis, methodology, resources, software, writing-original draft, writing—review and editing; R.F.: data curation, formal analysis, methodology, resources, software, writing-review and editing; C.P.: data curation, methodology, investigation, writing-review \& editing; G.L.R.: data curation, methodology, investigation, writing-review and editing; G.G.: data curation, methodology, investigation, writing-review and editing; A.D.T.: data curation, methodology, investigation, writing-review and editing; M.B. (Marta Brambilla): data curation, methodology, investigation, writing-review and editing; M.O.: data curation, methodology, investigation, writing-review and editing; S.M.: data curation, investigation, writing-review and editing; T.B.: data curation, investigation, writing-review and editing; A.B.: writing-review and editing; G.M.: writing-review and editing; E.Z.: data curation, investigation, writing-review and editing; R.G.: data curation, investigation; E.G.G.: data curation; M.G.: data curation; G.S.: data curation; F.G.M.d.B.: supervision, writing-review and editing; M.C.G.: conceptualization, investigation, methodology, supervision, writing-review and editing; M.R.: conceptualization, data curation, formal analysis, methodology, resources, software, supervision, validation, visualization, writing-review and editing; A.L.G.P.: conceptualization, data curation, formal analysis, methodology, resources, software, supervision, validation, visualization, writing-review and editing; F.T.: conceptualization, data curation, formal analysis, methodology, resources, software, supervision, validation, visualization, writing-review and editing. All authors have read and agreed to the published version of the manuscript. 
Funding: This research received no external funding.

Institutional Review Board Statement: The study was conducted according to the guidelines of the Declaration of Helsinki, and approved by Ethics Committee of Istituto Nazionale Tumori of Milan (protocol code INT22_15 and date of approval 17 January 2020).

Informed Consent Statement: Informed consent was obtained from all subjects involved in the study.

Data Availability Statement: Data are available from the authors upon reasonable request. The code for all the ML procedures is available at https:/ / trovo.faculty.polimi.it/downloads.html (accessed on 10 October 2021).

Acknowledgments: This work was supported by grants from AIRC (Asso-ciazione Italiana per la Ricerca sul Cancro): MFAG 2020 Rif. 25142-title: “Cancer Cell-Intrinsic PD-1 and immunemicroenvironment boost tumor growth upon immune-checkpoint inhibitors in NSCLC" PI Giuseppe Lo Russo. We acknowledge Anna Maria Leone, our nurse for assisting us with the patients included in this study.

Conflicts of Interest: The authors declare the following financial interests/personal relationships that may be considered as potential competing interests. M.C.G. declares personal financial interests with the following organizations: AstraZeneca, MSD International GmbH, BMS, Boehringer Ingelheim Italia S.p.A, Celgene, Eli Lilly, Ignyta, Incyte, Inivata, MedImmune, Novartis, Pfizer, Roche, Takeda. F.G.M.d.B. declares Consultant Advisory Board for Ignyta, BMS, Daiichi Sankyo, Pfizer, Octimet Oncology, Incyte, Teofarma, Pierre Fabre, Roche, EMD Serono, Sanofi, NMS Nerviano Medical Science, Pharm Research Associated (U.K) Ltd.; as a Speaker for BMS, Roche, MSD, Ignyta, Bayer, ACCMED, Dephaforum S.r.l., Nadirex, Merck, Biotechspert Ltd., PriME Oncology, Pfizer, Servier, Celgene, Tesaro, Loxo Oncology Inc., Sanofi, Healthcare Research \& Pharmacoepidemiology, as P.I for Novartis, Roche, BMS, Celgene, Incyte, NMS, Merck KGAA, Kymab, Pfizer, Tesaro, MSD. A.P. declares personal fees from Roche, AstraZeneca and BMS outside the submitted work. C.P. declares personal fees from BMS and MSD, outside the submitted work. G.L.R. declares personal fees from BMS, MSD and Astra Zeneca outside the submitted work. D.S. declares personal fees from AstraZeneca, Boehringer Ingelheim and BMS, outside the submitted work. The other authors report no conflict of interest.

\section{Abbreviations}

Non-small cell lung cancer (NSCLC), immunotherapy (IO), programmed death-ligand 1 (PD-L1), tumor mutational burden (TMB), tumor microenvironment (TME), microRNA (miRNA), artificial intelligence (AI), machine learning (ML), plasma microRNA signature classifier (MSC), quantitative reverse transcription PCR (RT-qPCR), low (L), intermediate (I), high $(\mathrm{H})$, hemolyzed (E), intravenously (IV), weeks (w); total body computed tomography (TB-CT), response evaluation criteria in solid tumors (RECIST1.1), complete response (CR), partial response (PR), stable disease (SD), progressive disease (PD), hyper progression disease (HPD), responder (R), non-responder (NR), median progression-free survival (mPFS), median overall survival (mOS), hazard ratio (HR), Akaike information criterion (AIC), neutrophil to lymphocyte ratio (NLR), lactate dehydrogenase (LDH), accuracy (ACC), area under the curve ROC (AUC), tumor-infiltrating lymphocyte (TIL). 


\section{Appendix A}

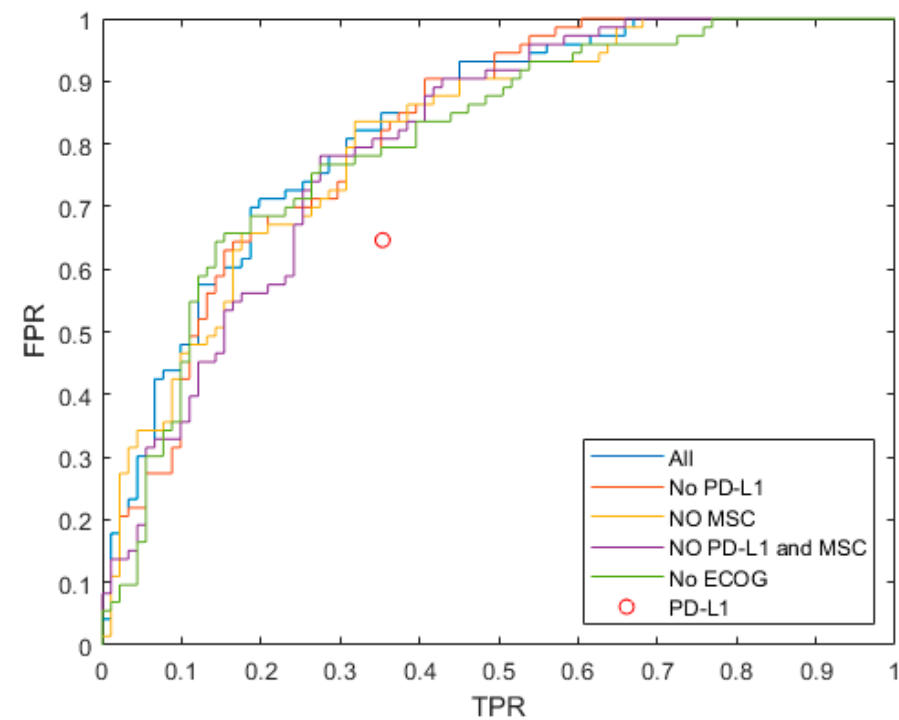

Figure A1. ROC curves for the LR method starting from different sets of features. The performance of PD-L1 is represented as a red circle. As suggested by the AUC confidence intervals, there is no method that outperforms the others significantly.

\section{A}
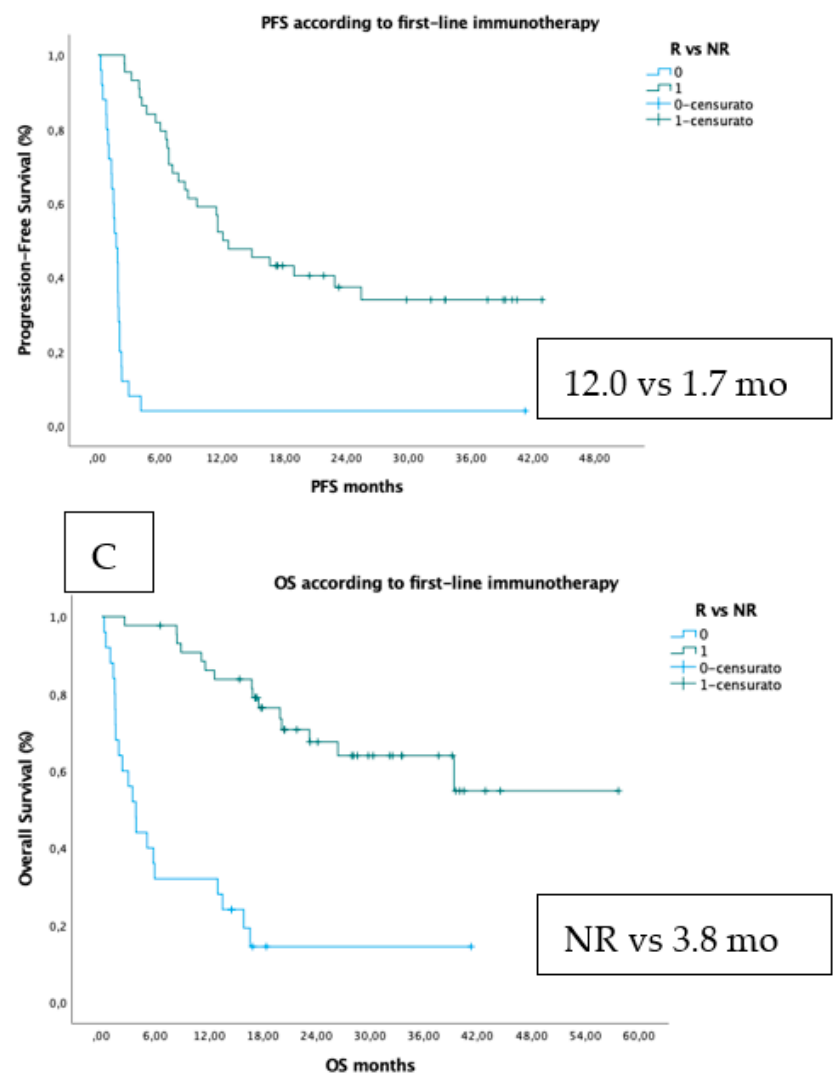

B
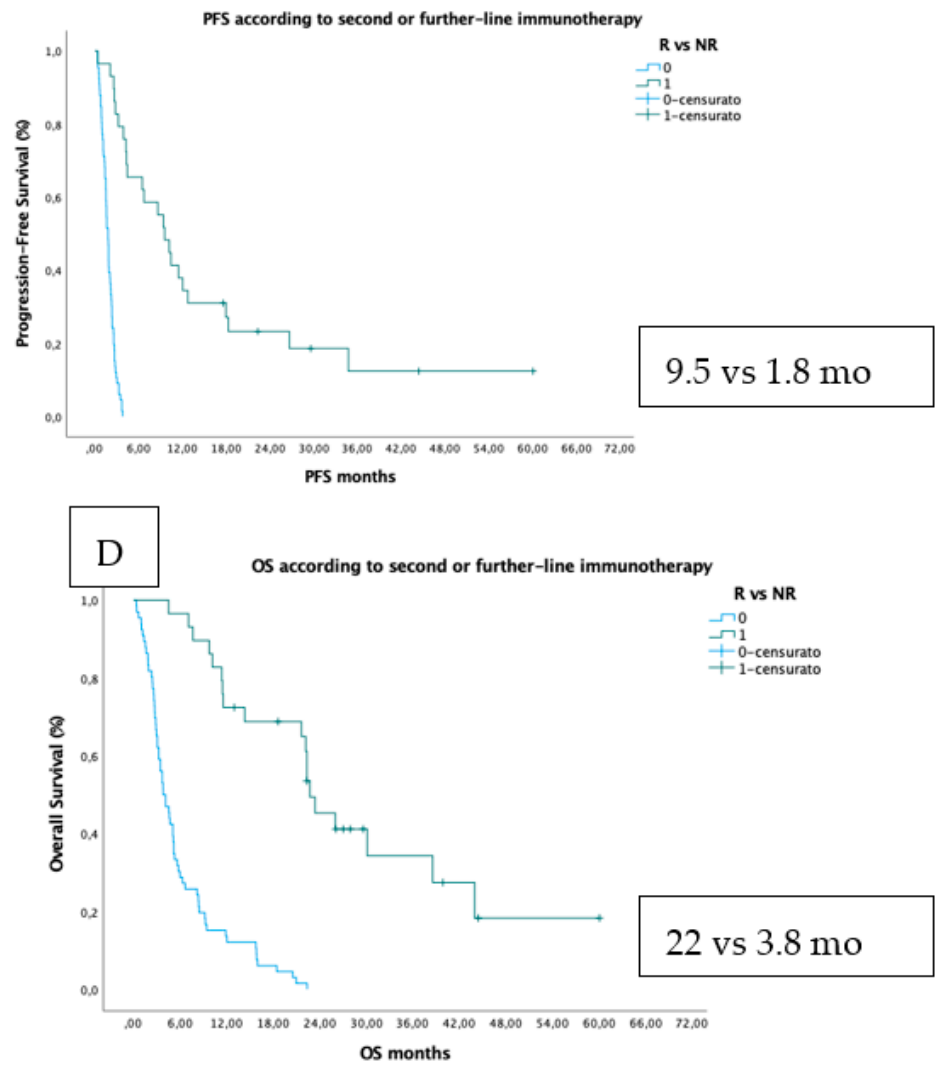

Figure A2. The Kaplan-Meier curves according to line of therapy in responder (R) and non-responder (NR) groups are reported. PFS for first (A) and second or further line (B) and OS for first (C) and second or further line IO (D). 


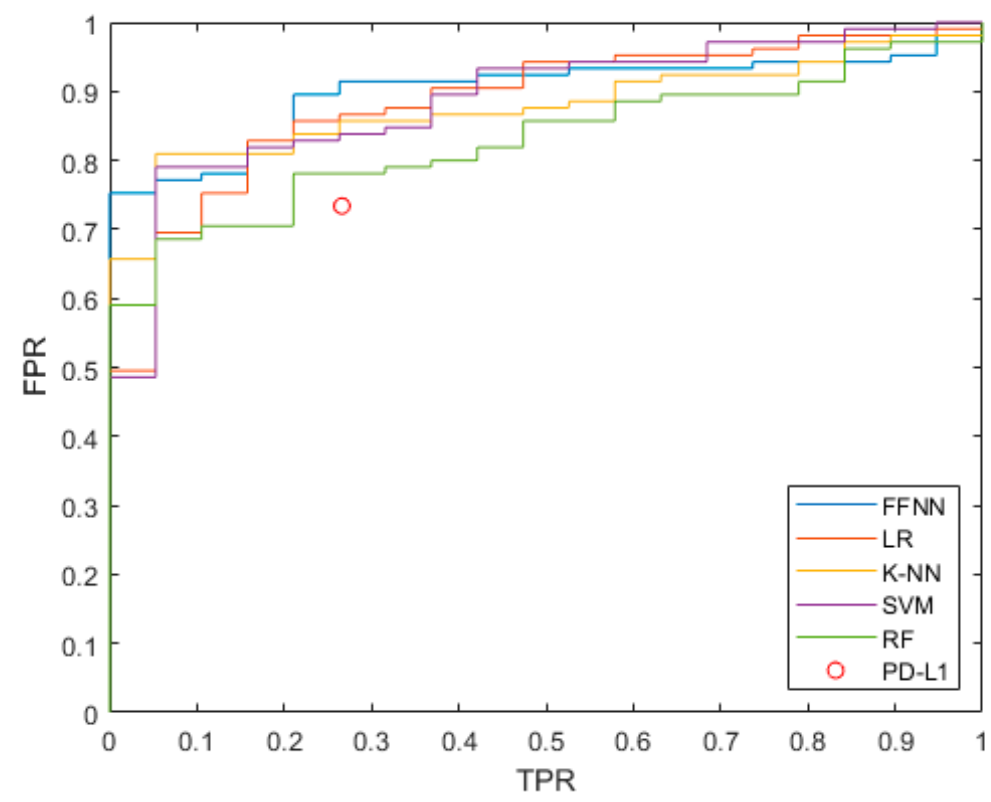

Figure A3. ROC curves for the LR method predicting the patients' survival (longer or shorter than 24 months). The performance of PD-L1 is represented as a red circle.

\section{References}

1. Sung, H.; Ferlay, J.; Siegel, R.L.; Laversanne, M.; Soerjomataram, I.; Jemal, A.; Bray, F. Global Cancer Statistics 2020: GLOBOCAN Estimates of Incidence and Mortality Worldwide for 36 Cancers in 185 Countries. CA Cancer J. Clin. 2021, 71, 209-249. [CrossRef]

2. $\quad$ Planchard, D.; Popat, S.; Kerr, K.; Novello, S.; Smit, E.F.; Faivre-Finn, C.; Mok, T.S.; Reck, M.; van Schil, P.E.; Hellmann, M.D.; et al. Correction to: "Metastatic non-small cell lung cancer: ESMO Clinical Practice Guidelines for diagnosis, treatment and follow-up". Ann. Oncol. 2019, 30, 863-870. [CrossRef]

3. Gettinger, S.; Horn, L.; Jackman, D.; Spigel, D.; Antonia, S.; Hellmann, M.; Powderly, J.; Heist, R.; Sequist, L.V.; Smith, D.C.; et al. Five-Year Follow-Up of Nivolumab in Previously Treated Advanced Non-Small-Cell Lung Cancer: Results From the CA209-003 Study. J. Clin. Oncol. 2018, 36, 1675-1684. [CrossRef]

4. $\quad$ Reck, M.; Rodríguez-Abreu, D.; Robinson, A.G.; Hui, R.; Csőszi, T.; Fülöp, A.; Gottfried, M.; Peled, N.; Tafreshi, A.; Cuffe, S.; et al. Updated Analysis of KEYNOTE-024: Pembrolizumab Versus Platinum-Based Chemotherapy for Advanced Non-Small-Cell Lung Cancer With PD-L1 Tumor Proportion Score of 50\% or Greater. J. Clin. Oncol. Off. J. Am. Soc. Clin. Oncol. 2019, 37, 537-546. [CrossRef]

5. Gadgeel, S.; Rodríguez-Abreu, D.; Speranza, G.; Esteban, E.; Felip, E.; Dómine, M.; Hui, R.; Hochmair, M.J.; Clingan, P.; Powell, S.F.; et al. Updated Analysis From KEYNOTE-189: Pembrolizumab or Placebo Plus Pemetrexed and Platinum for Previously Untreated Metastatic Nonsquamous Non-Small-Cell Lung Cancer. J. Clin. Oncol. 2020, 38, 1505-1517. [CrossRef]

6. Paz-Ares, L.; Vicente, D.; Tafreshi, A.; Robinson, A.; Soto Parra, H.; Mazières, J.; Hermes, B.; Cicin, I.; Medgyasszay, B.; RodríguezCid, J.; et al. A Randomized, Placebo-Controlled Trial of Pembrolizumab Plus Chemotherapy in Patients With Metastatic Squamous NSCLC: Protocol-Specified Final Analysis of KEYNOTE-407. J. Thorac. Oncol. 2020, 15, 1657-1669. [CrossRef]

7. Pai-Scherf, L.; Blumenthal, G.M.; Li, H.; Subramaniam, S.; Mishra-Kalyani, P.S.; He, K.; Zhao, H.; Zhao, H.; Yu, J.; Paciga, M.; et al. FDA Approval Summary: Pembrolizumab for Treatment of Metastatic Non-Small Cell Lung Cancer: First-Line Therapy and Beyond. Oncologist 2017, 22, 1392-1399. [CrossRef]

8. Reck, M.; Schenker, M.; Lee, K.H.; Provencio, M.; Nishio, M.; Lesniewski-Kmak, K.; Sangha, R.; Ahmed, S.; Raimbourg, J.; Feeney, K.; et al. Nivolumab plus ipilimumab versus chemotherapy as first-line treatment in advanced non-small-cell lung cancer with high tumour mutational burden: Patient-reported outcomes results from the randomised, open-label, phase III CheckMate 227 trial. Eur J. Cancer 2019, 116, 137-147. [CrossRef]

9. Petitprez, F.; Meylan, M.; de Reyniès, A.; Sautès-Fridman, C.; Fridman, W.H. The Tumor Microenvironment in the Response to Immune Checkpoint Blockade Therapies. Front. Immunol. 2020, 11, 784. [CrossRef]

10. Fortunato, O.; Borzi, C.; Milione, M.; Centonze, G.; Conte, D.; Boeri, M.; Verri, C.; Moro, M.; Facchinetti, F.; Andriani, F.; et al. Circulating mir-320a promotes immunosuppressive macrophages M2 phenotype associated with lung cancer risk. Int. J. Cancer 2019, 144, 2746-2761. [CrossRef]

11. Prelaj, A.; Tay, R.; Ferrara, R.; Chaput, N.; Besse, B.; Califano, R. Predictive biomarkers of response for immune checkpoint inhibitors in non-small-cell lung cancer. Eur. J. Cancer 2019, 106, 144-159. [CrossRef] 
12. Sivan, A.; Corrales, L.; Hubert, N.; Williams, J.B.; Aquino-Michaels, K.; Earley, Z.M.; Benyamin, F.W.; Lei, Y.M.; Jabri, B.; Alegre, M.L.; et al. Commensal Bifidobacterium promotes antitumor immunity and facilitates anti-PD-L1 efficacy. Science 2015, 350, 1084-1089. [CrossRef]

13. Yoon, H.J.; Kang, J.; Park, H.; Sohn, I.; Lee, S.H.; Lee, H.Y. Deciphering the tumor microenvironment through radiomics in non-small cell lung cancer: Correlation with immune profiles. PLoS ONE 2020, 15, e0231227. [CrossRef]

14. Prelaj, A.; Rebuzzi, S.E.; Pizzutilo, P.; Bilancia, M.; Montrone, M.; Pesola, F.; Longo, V.; Del Bene, G.; Lapadula, V.; Cassano, F. EPSILoN: A Prognostic Score Using Clinical and Blood Biomarkers in Advanced Non- Small-cell Lung Cancer Treated With Immunotherapy. Clin. Lung Cancer 2020, 21, 365-377. [CrossRef]

15. Sorich, M.J.; Rowland, A.; Karapetis, C.S.; Hopkins, A.M. Evaluation of the Lung Immune Prognostic Index for Prediction of Survival and Response in Patients Treated with Atezolizumab for NSCLC: Pooled Analysis of Clinical Trials. J. Thorac. Oncol. 2019, 14, 1440-1446. [CrossRef]

16. Triberti, S.; Durosini, I.; Pravettoni, G. A “Third Wheel” Effect in Health Decision Making Involving Artificial Entities: A Psychological Perspective. Front. Public Health 2020, 8, 117. [CrossRef] [PubMed]

17. Boeri, M.; Verri, C.; Conte, D.; Roz, L.; Modena, P.; Facchinetti, F.; Calabrò, E.; Croce, C.M.; Pastorino, U.; Sozzi, G. MicroRNA signatures in tissues and plasma predict development and prognosisof computed tomography detected lung cancer. Proc. Natl. Acad. Sci. USA 2011, 108, 3713-3718. [CrossRef] [PubMed]

18. Sozzi, G.; Boeri, M.; Rossi, M.; Verri, C.; Suatoni, P.; Bravi, F.; Roz, L.; Conte, D.; Grassi, M.; Sverzellati, N.; et al. Clinical utility of a plasma-based miRNA signature classifier within computed tomography lung cancer screening: A correlative MILD trial study. J. Clin. Oncol. 2014, 32, 768-773. [CrossRef]

19. Boeri, M.; Milione, M.; Proto, C.; Signorelli, D.; Lo Russo, G.; Galeone, C.; Verri, C.; Mensah, M.; Centonze, G.; Martinetti, A.; et al Circulating miRNAs and PD-L1 Tumor Expression Are Associated with Survival in Advanced NSCLC Patients Treated with Immunotherapy: A Prospective Study. Clin. Cancer Res. 2019, 25, 2166-2173. [CrossRef]

20. Prelaj, A.; Proto, C.; Lo Russo, G.; Signorelli, D.; Ferrara, R.; Mensah, M.; Galli, G.; De Toma, A.; Viscardi, G.; Brambilla, M.; et al. Integrating clinical and biological prognostic biomarkers in patients with advanced NSCLC treated with immunotherapy: The DEMo score system. Transl Lung Cancer Res. 2020, 9, 617-628. [CrossRef]

21. Mensah, M.; Borzi, C.; Verri, C.; Suatoni, P.; Conte, D.; Pastorino, U.; Orazio, F.; Sozzi, G.; Boeri, M. MicroRNA Based Liquid Biopsy: The Experience of the Plasma miRNA Signature Classifier (MSC) for Lung Cancer Screening. J. Vis. Exp. 2017, 128, 56326. [CrossRef]

22. Ferrara, R.; Mezquita, L.; Texier, M.; Lahmar, J.; Audigier-Valette, C.; Tessonnier, L.; Mazieres, J.; Zalcman, G.; Brosseau, S.; Le Moulec, S.; et al. Hyperprogressive Disease in Patients With Advanced Non-Small Cell Lung Cancer Treated With PD-1/PD-L1 Inhibitors or With Single-Agent Chemotherapy. JAMA Oncol. 2018, 4, 1543-1552. [CrossRef] [PubMed]

23. Lo Russo, G.; Moro, M.; Sommariva, M.; Cancila, V.; Boeri, M.; Centonze, G.; Ferro, S.; Ganzinelli, M.; Gasparini, P.; Huber, V.; et al. Antibody-Fc/FcR Interaction on Macrophages as a Mechanism for Hyperprogressive Disease in Non-small Cell Lung Cancer Subsequent to PD-1/PD-L1 Blockade. Clin. Cancer Res. 2019, 25, 989-999. [CrossRef]

24. NSCLC Response Prediction to IO Using ML Methods. Available online: https://trovo.faculty.polimi.it/downloads.html (accessed on 10 October 2021).

25. He, B.; Dong, D.; She, Y.; Zhou, C.; Fang, M.; Zhu, Y.; Zhang, H.; Huang, Z.; Jiang, T.; Tian, J.; et al. Predicting response to immunotherapy in advanced non-small-cell lung cancer using tumor mutational burden radiomic biomarker. J. Immunother. Cancer 2020, 8, 1-10. [CrossRef]

26. Khorrami, M.; Prasanna, P.; Gupta, A.; Patil, P.; Velu, P.D.; Thawani, R.; Corredor, G.; Alilou, M.; Bera, K.; Fu, P.; et al. Changes in CT Radiomic Features Associated with Lymphocyte Distribution Predict Overall Survival and Response to Immunotherapy in Non-Small Cell Lung Cancer. Cancer Immunol. Res. 2020, 8, 108. [CrossRef] [PubMed]

27. Yang, Y.; Yang, J.; Shen, L.; Chen, J.; Xia, L.; Ni, B.; Ge, L.; Wang, Y.; Lu, S. A multi-omics-based serial deep learning approach to predict clinical outcomes of single-agent anti-PD-1/PD-L1 immunotherapy in advanced stage non-small-cell lung cancer. Am. J. Transl. Res. 2021, 13, 743-756.

28. Fang, C.; Xu, D.; Su, J.; Dry, J.R.; Linghu, B. DeePaN: Deep patient graph convolutional network integrating clinico-genomic evidence to stratify lung cancers for immunotherapy. NPJ Digital Med. 2021, 4, 14. [CrossRef]

29. Tian, P.; He, B.; Mu, W.; Liu, K.; Liu, L.; Zeng, H.; Liu, Y.; Jiang, L.; Zhou, P.; Huang, Z.; et al. Assessing PD-L1 expression in non-small cell lung cancer and predicting responses to immune checkpoint inhibitors using deep learning on computed tomography images. Theranostics 2021, 11, 2098-2107. [CrossRef]

30. Xue, L.; Bi, G.; Zhan, C.; Zhang, Y.; Yuan, Y.; Fan, H. Development and Validation of a 12-Gene Immune Relevant Prognostic Signature for Lung Adenocarcinoma Through Machine Learning Strategies. Front. Oncol. 2020, 10, 1-14. [CrossRef] [PubMed]

31. Lai, Y.H.; Chen, W.N.; Hsu, T.C.; Lin, C.; Tsao, Y.; Wu, S. Overall survival prediction of non-small cell lung cancer by integrating microarray and clinical data with deep learning. Sci. Rep. 2020, 10, 4679. [CrossRef]

32. Bartholomai, J.A.; Frieboes, H.B. Lung Cancer Survival Prediction via Machine Learning Regression Classification, and Statistical Techniques. Proc. IEEE Int. Symp. Signal Proc. Inf. Tech. 2018. [CrossRef]

33. Johannet, P.; Coudray, N.; Donnelly, D.M.; Jour, G.; Illa-Bochaca, I.; Xia, Y.; Johnson, D.B.; Wheless, L.; Patrinely, J.R.; Nomikou, S.; et al. Using Machine Learning Algorithms to Predict Immunotherapy Response in Patients with Advanced Melanoma. Clin. Cancer Res. 2021, 27, 131-140. [CrossRef] [PubMed] 
34. Lu, Z.; Chen, H.; Jiao, X.; Zhou, W.; Han, W.; Li, S.; Liu, C.; Gong, J.; Li, J.; Zhang, X.; et al. Prediction of immune checkpoint inhibition with immune oncology-related gene expression in gastrointestinal cancer using a machine learning classifier. J. Immunother. Cancer 2020, 8, 1-5. [CrossRef] [PubMed]

35. Rundo, F.; Spampinato, C.; Banna, G.L.; Conoci, S. Advanced deep learning embedded motion radiomics pipeline for predicting anti-PD-1/PD-L1 immunotherapy response in the treatment of bladder cancer: Preliminary results. Electronics 2019, 8, 1134. [CrossRef] 